Article

\title{
Energy Reduction Multipath Routing Protocol for MANET Using Recoil Technique
}

\author{
Rakesh Kumar Sahu * and Narendra S. Chaudhari \\ Department of Computer Science \& Engineering, Indian Institute of Technology, Indore 453552, India; \\ nsc0183@yahoo.com \\ * Correspondence: rekeshsahubl@yahoo.co.in; Tel.: +91-789-851-4607
}

Received: 15 March 2018; Accepted: 23 April 2018; Published: 25 April 2018

\begin{abstract}
In Mobile Ad-hoc networks (MANET), power conservation and utilization is an acute problem and has received significant attention from academics and industry in recent years. Nodes in MANET function on battery power, which is a rare and limited energy resource. Hence, its conservation and utilization should be done judiciously for the effective functioning of the network. In this paper, a novel protocol namely Energy Reduction Multipath Routing Protocol for MANET using Recoil Technique (AOMDV-ER) is proposed, which conserves the energy along with optimal network lifetime, routing overhead, packet delivery ratio and throughput. It performs better than any other AODV based algorithms, as in AOMDV-ER the nodes transmit packets to their destination smartly by using a varying recoil off time technique based on their geographical location. This concept reduces the number of transmissions, which results in the improvement of network lifetime. In addition, the local level route maintenance reduces the additional routing overhead. Lastly, the prediction based link lifetime of each node is estimated which helps in reducing the packet loss in the network. This protocol has three subparts: an optimal route discovery algorithm amalgamation with the residual energy and distance mechanism; a coordinated recoiled nodes algorithm which eliminates the number of transmissions in order to reduces the data redundancy, traffic redundant, routing overhead, end to end delay and enhance the network lifetime; and a last link reckoning and route maintenance algorithm to improve the packet delivery ratio and link stability in the network. The experimental results show that the AOMDV-ER protocol save at least $16 \%$ energy consumption, $12 \%$ reduction in routing overhead, significant achievement in network lifetime and packet delivery ratio than Ad hoc on demand multipath distance vector routing protocol (AOMDV), Ad hoc on demand multipath distance vector routing protocol life maximization (AOMR-LM) and Source routing-based multicast protocol (SRMP) algorithms. Hence, the AOMDV-ER algorithm performs better than these recently developed algorithms.
\end{abstract}

Keywords: link error; mobile ad hoc network; network lifetime; on demand routing; residual energy

\section{Introduction}

Mobile ad hoc network (MANET) is infrastructureless wireless type network in which the packet transmission from one node to another takes place without any access point. Therefore, MANET can be deployed in hostile and difficult situations where a wired network is not advisable or possible. Furthermore, it is deployed in adverse situations, where battery replacement in any node is not feasible. Thus, routing protocol plays a pivotal role while forwarding the packet in the network. Moreover, it is necessary to reduce the number of failed transmissions, data redundancies and traffic overhead of mobile nodes, which results in the reduction of energy consumption substantially. The Ad hoc network has many features such as easy deployment, when and where need arises, and hence it is gaining popularity in widespread applications such as in military battlefields to establish information networks 
amongst the soldiers, weapon systems, command control center and vehicles (Figure 1). The ad hoc network architecture is being used in many real time business applications in order to increase the efficiency and effectiveness along with a level of profit optimization at large in the corporate companies. The applications are spread in all dimensions of life and covers many scenarios and services via tactical networks, emergency services, civil/commercial arena, home and enterprise environment, education system, entertainment, sensor network and context aware services (call forwarding, location specific information, time dependent services, etc.). However, there are many challenges which require the attention of research to plug them in efficient manner, such as frequent link failure among the nodes (due to random and dynamic behavior), no central administration without infrastructure, being susceptible to attack, inordinate consumption of power (energy constraint operation), limited bandwidth, network scalability, device heterogeneity, muti-hop routing, self-creation, self-organization and self-administration. The acute problem related to energy consumption and link failure in networks has drawn the attention of many researchers and scientists which led to the development of various tools and techniques based on transmission energy consumption, residual energy consumption or both. One recent advancement in the arena of routing protocol is multipath routing protocol. Still, there are certain issues in multipath routing protocols, one of them is energy consumption in the discovery of an optimal path, rediscovery of path when link failure encounters and retransmission of packets if required. Route discovery involves a reasonable amount of energy when network is small. Subsequently, when the size of the network grows, then route discovery demands huge energy and becomes a problem of sustaining the network for long time, as the gigantic portion of energy is consumed in route discovery itself. As in paper [1], many energy-aware packet forwarding routing protocol have been developed based on the criteria of link cost, link error, residual energy. The on-demand energy-based forwarding strategy (OEFS) enables the node to consume less energy but the route selection criterion is not based on link stability and leads to frequent link error [2]. Further work was extended in FF-AOMDV [3], which is based on calculation of fitness function, while energy level and number of hop count are taken into consideration from source to destination before sending the packets. In the proposed AOMDV-ER protocol the foremost objectives are reduce the power consumption of route discovery, retransmission overhead and transmission failure (due to link failure). All these three issues conserve the battery power for subsequent fruitful transmission of packets, which leads to a better network lifetime.

The rest of the paper is organized as follows. Section 2 focuses on previous work that has been done in this area; Section 3, the proposed work, broadly consists of three subparts. Section 4 expresses the mathematical support for the result demonstration and Section 5 demonstrates the experimental result of proposed protocol. The final Section 6 conveys the conclusion of proposed work.

\section{Review of the State of the Art and Main Contributions of the Work}

The work in FF-AOMDV [3] is based on the calculation of the fitness function, where energy level and hop count are taken into consideration, between source and destination, before sending the packets. However, each of AOMDV, AOMR-LM and FF-AOMDV has a few shortcomings. For example, AOMDV requires the least energy of the three but throughput and end to end delay was degraded. AOMR-LM has the least end to end delay and a consumption of energy was achieved but also demonstrated the worst performance in packet delay ratio. Protocol FF-AOMDV addressed the problem encountered in discussed protocols, but could not resolve the problem of network lifetime [3]. In Ad-Hoc network, packet transmission is a critical issue which was developed with geographic positioning protocols [4]. Buffer management is taken into consideration [5] as well as the multipath delivery scheme [6]. In the paper [7], the author has proposed an effective position based opportunistic protocol founded on the geographic routing based stateless and the nature of the transmission. This protocol focuses on virtual destination to avoid the communication holes. A multicast data transmission scheme [8] was used in multi-hop wireless networking that avoids support routing cycles or packet duplication. The author presented a table to avoid packet duplication in the routing protocol. 
It has reached a high speed packet delivery as compared to mesh-based routing. The robust and secure routing scheme is proposed in [9] for highly dynamic ad hoc networks for calculating disjoined path nodes. The author has observed the reliability and security of the path to obtain a highly secure network routing. Based on the information of node location, the transmission overhead had been decreased during path discovery and maintenance. In this path, network progression is not taken into consideration. Based on security improvement mechanism, a secure end-to-end packet delivery was achieved [6]. A stable multicast routing path was established from source to destination expressed in mesh-based multicast routing mechanism [10]. In this work, nodes, which have high stable links, joined together to construct the stable path. In [11], the author has proposed two schemes via a cluster based approach and location based approach, to avoid traffic and define each area of cluster. The relationship between mobile nodes and the appropriate size of the clustering area was determined. The Opportunistic Routing and Encoding based on MAC-independent [12], was introduced to improve reliable communication. Both location information and transmission probability were used for efficient packet distribution. In this scheme, when information updating occurs, each node in the network can have the latest information up to two hop neighbors, when they are updated periodically. A new Improved Distribution Channel Access scheme based on contention algorithm and hub control [13] provides probabilistic and combined QoS support to regulate the controversial window. This scheme offers a traffic differentiation. In [14], a routing scheme based on the stability of the route measurements and residual energy during detection is presented. The estimation of link-connection stability was made based on the received signal strength. Based on location, a new routing scheme is proposed in [15] to reduce the consumption energy. In [16], the Hybrid Delivery Network content system has been proposed to combine routers with the new content overlay server. In [17], the On Demand Multicast Routing Protocol has been developed to build routes and keeps membership of multicast groups. However, the stability of the routes is being overlooked. In the intended approach, the protocol can endure all attacks and environmental defects. In [18], an improved version of topological multicast routing protocol's performance analysis has been proposed. The author formulated a function based on route length and route energy to optimize the values of consumed energy using the binary particle swarm optimization (SWO) algorithm. The on-demand energy-based forwarding strategy (OEFS) enables the nodes to consume less energy but the route selection criterion is not based on reliability [2]. In [19], a protocol based on energy efficient routing was proposed, named AOMR-LM, which is an improvement on AOMDV [20].

Our approach discovers the route in such a way that it can reduce data redundancy and minimize the traffic overhead, which aids in the power conservation and leads to the enhancement of network life time. The concept of recoiled nodes further reduces the routing overhead whereas the last part of algorithm, namely the link reckoning algorithm, ensures break free packet transmission, lowers the packet loss and protects from inordinate delay in the network. Details of protocol are given in subsequent section of the paper.

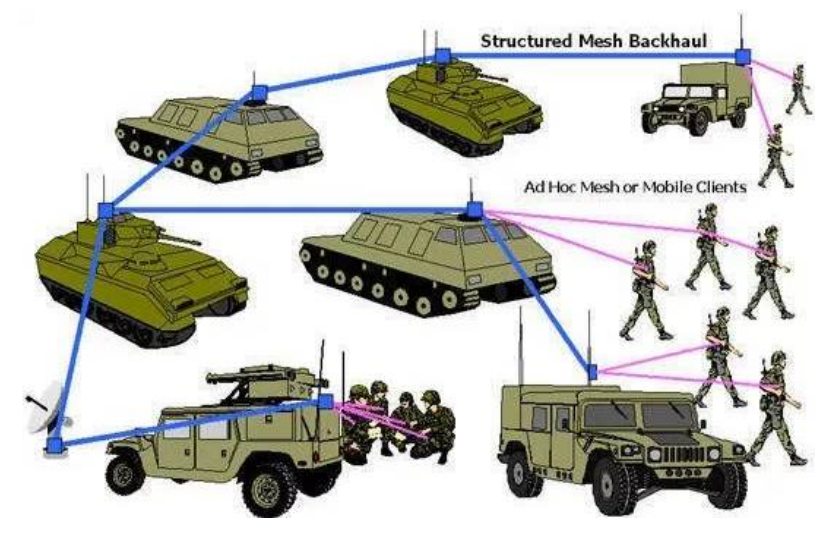

Figure 1. Illustrates the application of MANET in tactical network. 


\section{Proposed Protocol (AOMDV-ER)}

Some recently proposed algorithms, namely AOMR-LM, FF-AOMDV [3] and AOMDV, have some shortcomings. The AOMDV and AOMR-LM have the least end to end delay and consumption of energy but demonstrated the worst performance in packet delay ratio. Whereas the other protocol FF-AOMDV addressed the problem encountered in the AOMR-LM protocols, but could not resolve the problem of network lifetime [3]. These algorithms did not consider the node battery level evaluation and link strength estimation before making them (nodes) as the part of the route. However, the proposed algorithm AOMDV-ER has given due weight to this parameter.

The Energy Reduction Multipath Routing Protocol for MANET using Recoil Technique (AOMDV-ER), is the improvement over the well-known ad hoc on demand distance vector routing (AODV), and ad hoc on demand multipath distance vector routing AOMDV, AOMR-LM and FF-AOMDV. The proposed routing consists of three optimized algorithms via Optimal route discovery; coordinated recoiled node algorithm, to reduce the further reduction in battery power; and the last algorithm takes care of the route maintenance efficiently, to reduce the chances of retransmission, which is an important provision for saving the energy. The description is expressed in the subsequent section.

\subsection{Optimal Route Discovery Algorithm}

The algorithm is based on residual energy and geographical location which uses the recoil technique. The link cost of each route is evaluated on the basis of node distance from SD line. The least value is assigned to the node nearer to the SD line. This optimal route discovery algorithm is depicted in Algorithm 1.

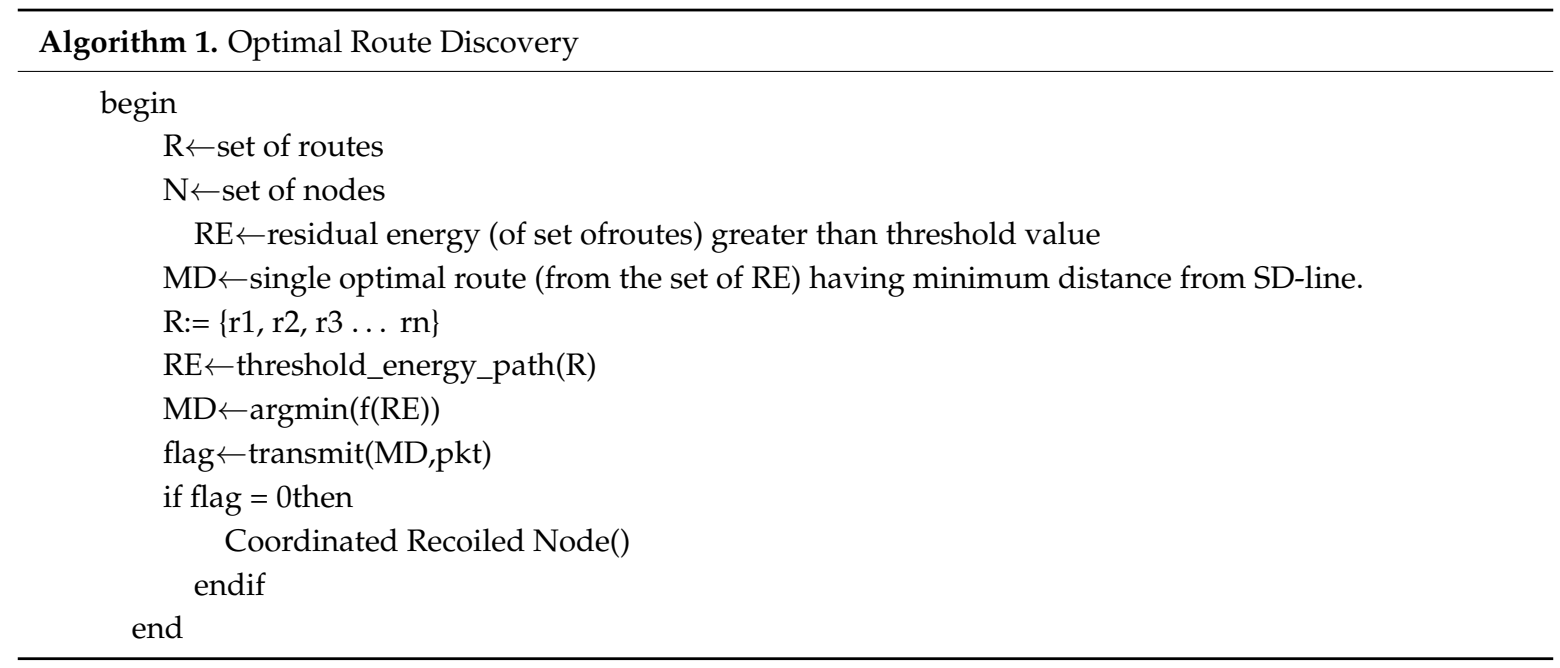

Given S: source and D: (Figure 2a,b) destination. $(S \rightarrow B \rightarrow D)(S \rightarrow C \rightarrow D)$ and $(S \rightarrow A \rightarrow D)$ are probable routes from $S$ to $D$. The minimum consumes energy or minimum route cost is $(\mathrm{S} \rightarrow \mathrm{B} \rightarrow \mathrm{D})$. However, the optimized path is based on the max-min metric, which is $(\mathrm{S} \rightarrow \mathrm{A} \rightarrow \mathrm{D})$. Figure 3, describes the optimal route on the basis of distance from the line joining $S$ and $D$. The minimum summation of perpendicular distance carrying path would be the optimal path. 
Link cost

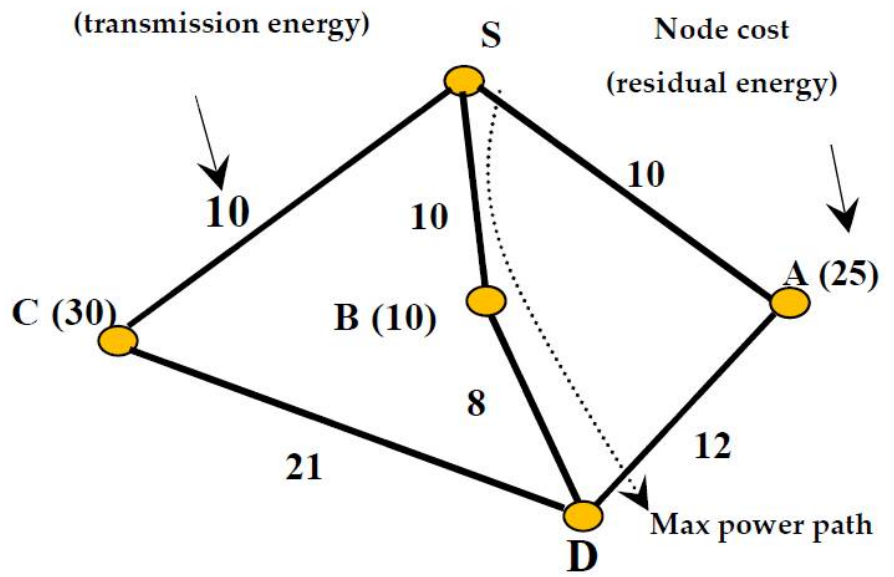

(a)

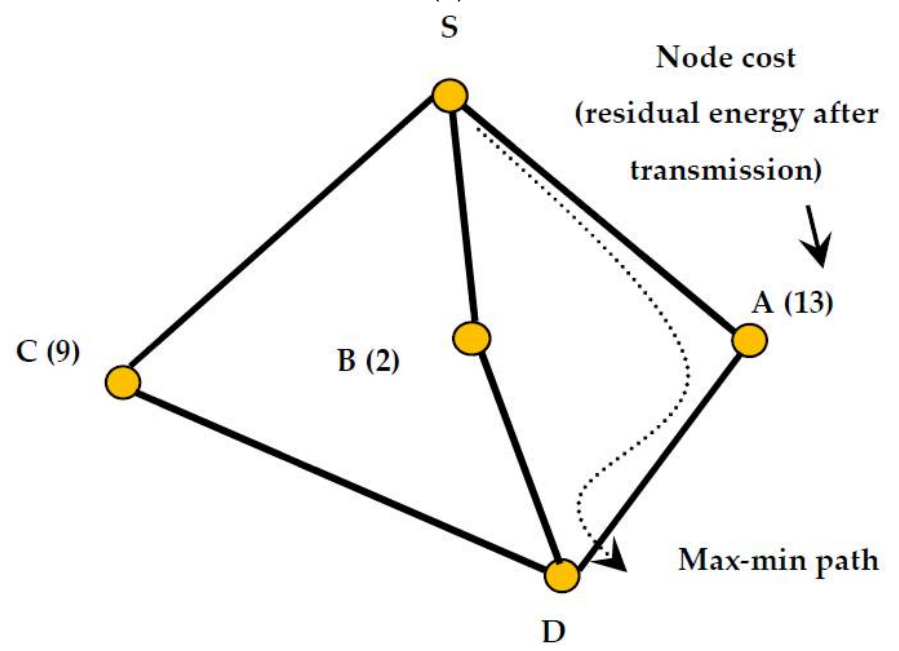

(b)

Figure 2. (a) Min-power route; (b) Max-Min Energy Distance Path.

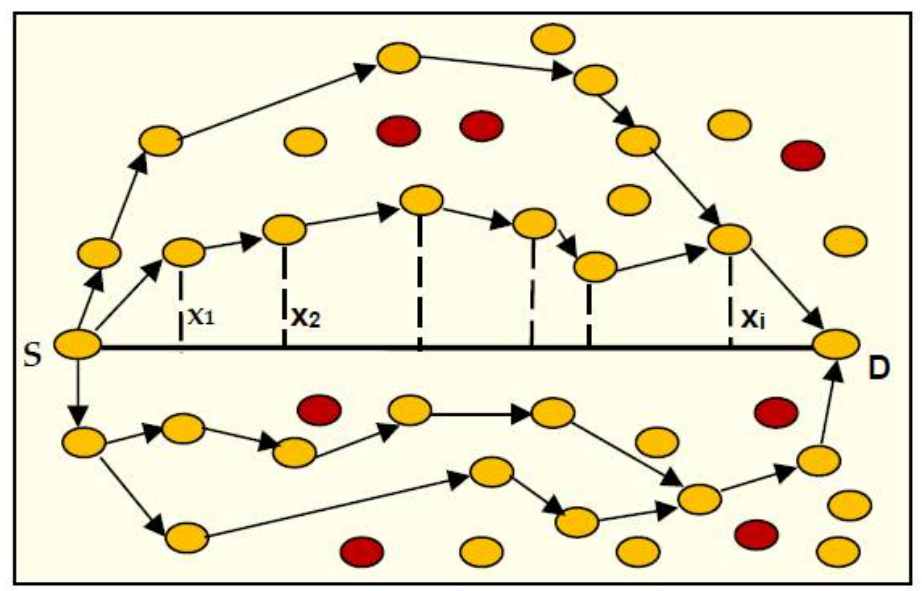

$\bigcirc$ Trustable node $\bigcirc$ Non-trustable node

Figure 3. Minimum distance from SD line. 


\subsection{Coordinated Recoiled Node Algorithm}

In routing, usually a node floods the packet to its neighborhood and this process continues till the packet reaches its destination. However, in the proposed approach, instead of all nodes transmitting the packet, only a few eligible nodes participate in transmission. These specific nodes are called recoiled nodes and the selection of recoiled nodes is made as per the algorithm based on node's location illustrated below.

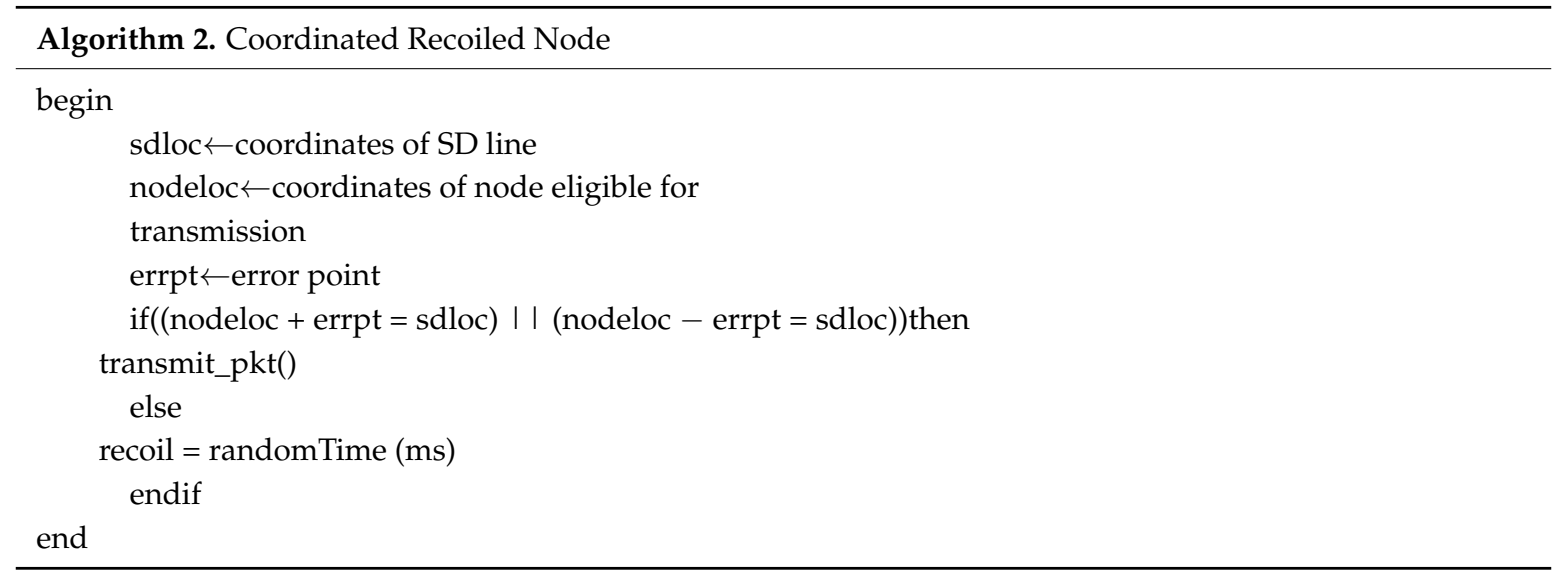

As the algorithm 2 expresses, only very few of them are eligible to further transmit the packet and the remaining retain the packet with them as transmission requires more power than sensing the network. Figure 4, shows the same strategy: node $\mathrm{S}$ transmits the packet to node A, B, C and node D. Further, out of these four nodes, only two eligible nodes (node B and C) can transmit the packet to their neighbors and the remaining nodes (node A and E) wait for their eligibility called recoil nodes. The eligibility of a node is based on the eligibility function of variable location from the SD line.

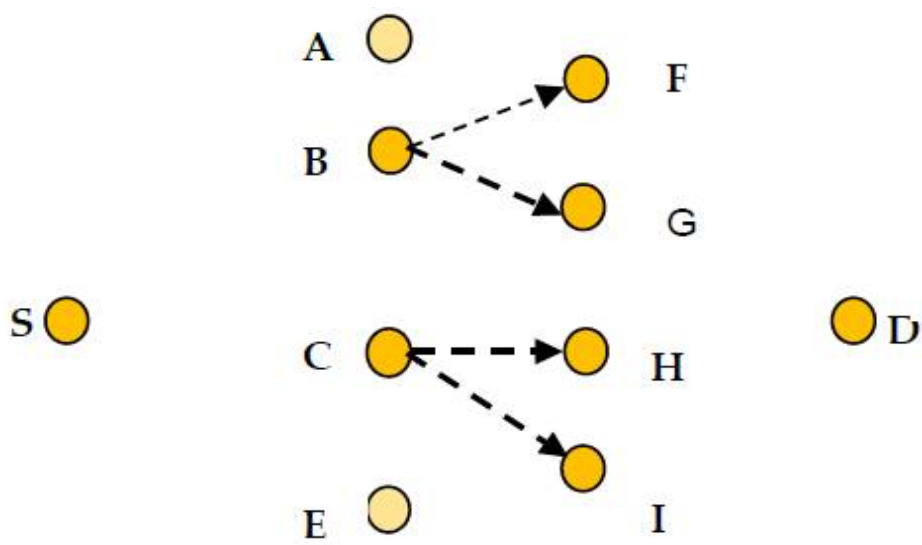

Figure 4. Recoil technique with recoiled nodes.

The nodes which are on the SD line transmit and those which are away from the SD line will be assigned variable recoil time. Figure 5 a, shows recoil time (waiting time) to node1, 2 and 3 as per the location, i.e., node $1<$ node $2<$ node 3 . So, lower valued recoil time nodes turn out to be part of primary routes. However, if any point of time transmission is failed (due to any reason like packet loss, link error) then retransmission by the recoiled becomes inevitable. The prediction of link error is address in subsequent section. 


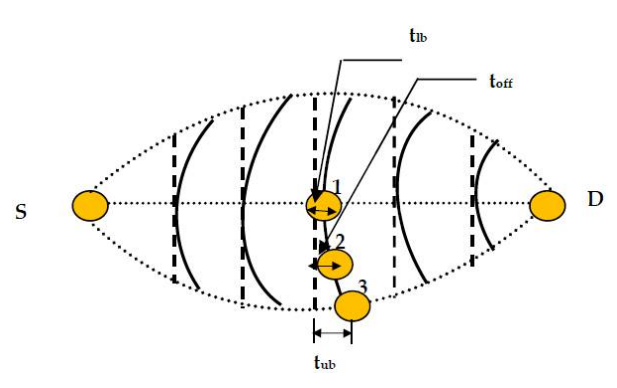

(a)

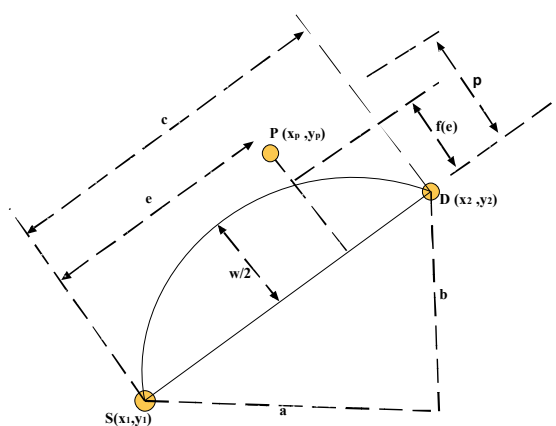

(b)

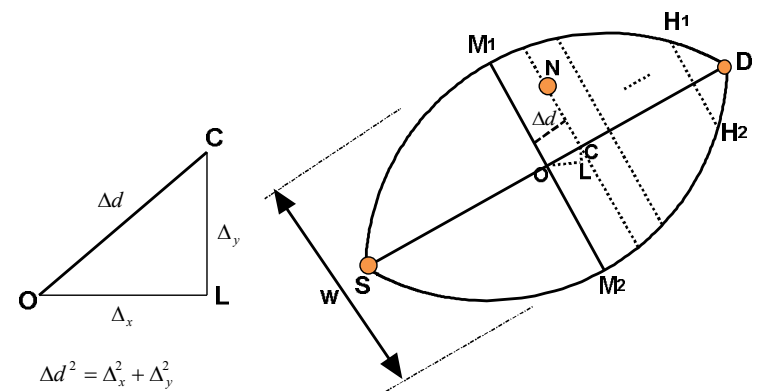

(c)

Figure 5. (a) Recoiled off time distribution to nodes 1, 2 and 3; (b) Recoiled off time calculation; (c) Recoiled off time calculation for an arbitrary node $\mathrm{N}$.

\subsection{Computation of Recoil off time}

The Figure $5 \mathrm{~b}$ shows the recoil time calculation to nodes which are inside the curve. First, it is necessary to find out whether point $P\left(x_{p}, y_{p}\right)$ is inside the curve or not. The calculation related to this carried out as follows

$$
c^{2}=a^{2}+b^{2}, a=x_{2}-x_{1}, b=y_{2}-y_{1}
$$

The slope of SD line $m=b / a$ and the equation can be written as

$$
\begin{gathered}
y-y_{1}=m\left(x-x_{1}\right) \\
y-y_{1}=\frac{b}{a}\left(x-x_{1}\right) \\
b x-a y-\left(b x_{1}-a y_{1}\right)=0
\end{gathered}
$$

The perpendicular distance of $P\left(x_{p}, y_{p}\right)$ from SD line can be express as under

$$
p=\frac{\left|b x_{p}-a y_{p}-b x_{1}+a y_{1}\right|}{\sqrt{\left(a^{2}+b^{2}\right)}}=\frac{\left|a\left(y_{1}-y_{p}\right)-b\left(x_{1}-x_{p}\right)\right|}{c}
$$

The equation of line perpendicular to SD line and passing through point $P$ can be given as

$$
\begin{gathered}
y-y_{p}=-\frac{a}{b}\left(x-x_{p}\right) \\
a x+b y-\left(a x_{p}+b y_{p}\right)=0
\end{gathered}
$$

The value of e is the perpendicular distance on line (4) from point $S$.

$$
e=\frac{\left|a x_{1}+b y_{1}-\left(a x_{p}+b y_{p}\right)\right|}{c}
$$




$$
e=\frac{a\left(x_{p}-x_{1}\right)+b\left(y_{p}-y_{1}\right)}{c}
$$

The shape of the curve is approximately an ellipse, hence the function $f(e)$, which gives the extreme value for point $P$ in order to be inside the ellipse. The center coordinate $\left(C_{1}\right)$ of an ellipse, equation of an ellipse and value of the $f(e)$ can be calculated as below.

$$
\mathrm{C}_{1}\left(\frac{x_{1}+x_{2}}{2}, \frac{y_{1}+y_{2}}{2}\right)
$$

Equation of an ellipse

$$
\begin{gathered}
\frac{\left[x-\frac{\left(x_{1}+x_{2}\right)}{2}\right]^{2}}{\left(\frac{c}{2}\right)^{2}}+\frac{\left[y-\frac{\left(y_{1}+y_{2}\right)}{2}\right]^{2}}{\left(\frac{w}{2}\right)^{2}}=1 \\
f(e)=\left\{\begin{array}{c}
0 \text { when } \alpha=0 \\
\sqrt{\alpha * \beta^{2}} \text { otherwise }
\end{array}\right.
\end{gathered}
$$

where $\alpha=1-\left[\frac{\left(e-\frac{c}{2}\right)^{2}}{\delta^{2}}\right], \beta=\frac{w}{2}$ and $\delta=\frac{c}{2}$

The recoil off time (for recoil nodes) is directly proportional to the magnitude of perpendicular distance from point $\mathrm{N}$ to SD line as shown in Figure 5c. It means recoil off time increases with the perpendicular distance on SD line. Let us consider an arbitrary recoiled node, say $\mathrm{N}$, at distance $\Delta d$ from $\mathrm{M}_{1} \mathrm{M}_{2}$ and the coordinate of point $\mathrm{C}\left(\frac{x_{1}+x_{2}+\Delta_{x}}{2}, \frac{y_{1}+y_{2}+\Delta_{y}}{2}\right)$. The equation of a line passing through point $\mathrm{N}$, can be expressed as given below in Equation (2b), after simplification, it results in Equation (9).

$$
\begin{aligned}
& b\left[y-\frac{\left(y_{1}+y_{2}+\Delta_{y}\right)}{2}\right]=-a\left[x-\frac{\left(x_{1}+x_{2}+\Delta_{x}\right)}{2}\right] \\
& 2 a x+2 b y-b\left(y_{1}+y_{2}+\Delta_{y}\right)-a\left(x_{1}+x_{2}+\Delta_{x}\right)=0
\end{aligned}
$$

In $\triangle \mathrm{CLO}$, the right-angled identity exists, given by Equation (10).

$$
\Delta d^{2}=\Delta_{x}^{2}+\Delta_{y}^{2}
$$

Additionally, the line passing through the center of the curve can be expressed by keeping $\Delta_{x}=0$ and $\Delta_{y}=0$ in Equation (8), (here parameter would be maximum $=\mathrm{w}$ )

$$
\begin{aligned}
& b\left[y-\frac{\left(y_{1}+y_{2}\right)}{2}\right]=-a\left[x-\frac{\left(x_{1}+x_{2}\right)}{2}\right] \\
& 2 a x+2 b y-b\left(y_{1}+y_{2}\right)-a\left(x_{1}+x_{2}\right)=0
\end{aligned}
$$

With the help of Equations (9) and (10), the value of recoil off time can be obtained for any node, and finally reduces to Equation (11).

\subsection{Link Reckoning and Route Maintenance Algorithm}

The link strength reckoning can be done before the link error occurs, which helps in ensuring the improved packet delivery ratio.

Consider $\mathrm{n}$ nodes between source and destination. The required transmission energy in each link can be express as $p_{i, i+1}=a s_{i . i+1}^{\alpha}$, Where $s i, i+1$ refers to link cost between nodes $i$ and $i+1$, $a$ is the environmental constant and $\alpha>2$. Each link in a route has an independent link error rate 
assuming $e_{i, i+1}$. The number of transmission between nodes $i$ and $i+1$ is a distributed random variable $X$ (geometrically), such that

$$
\operatorname{Prob}\{X=x\}=e_{i, i+1}^{x-1} \times\left(1-e_{i, i+1}\right) ; \forall x
$$

For the successful transfer of a packet, it is therefore required that $\frac{1}{\left(1-e_{i, i+1}\right)}$ is the mean number of transmission. Thus, the effective transmission energy between nodes $i$ and $i+1$, (including the effect of link error) is given by Equation (13)

$$
C_{i, i+1}=\frac{p_{i, i+1}}{\left(1-e_{i, i+1}\right)}=\frac{a s_{i, i+1}^{\alpha}}{\left(1-e_{i, i+1}\right)}
$$

The link error rate changes frequently, hence probability distributions with random variables have been taken into consideration, which is given by Equation (12) and the similar case is for the transmission energy between the two corresponding nodes. However, the prediction based link lifetime of a node can be estimated before the link error occurs between nodes, which are expressed by Equation (13).

$$
\begin{aligned}
& P_{r}=\frac{P_{T}}{4 \pi d_{i}^{2}} \\
& d_{i}^{2}=\frac{P_{T}}{4 \pi P_{r}}
\end{aligned}
$$

The distance and the transmission power are the determinant factors for computing the strength of the received signal. Consider the points $\mathrm{P}, \mathrm{A}, \mathrm{B}, \mathrm{E}$ and $\mathrm{M}$ as a few locations of node $\mathrm{P}$. The relative speed and the distance determines the link lifetime of node in network. The node $\mathrm{P}$ moves from $\mathrm{A}$ to $\mathrm{B}$ in $\Delta t_{2}$ time interval and from B to $\mathrm{E}$ in $\Delta t_{1}$ time interval $\left(\Delta t_{1}=\Delta t_{2}=\Delta t\right)$. Similarly, several such reference points are taken into consideration in order to determine the link lifetime and the relative speed. The relative speed can be express as follows with the help of Figure $6 \mathrm{~b}$, (using cosine rule)

$$
\begin{aligned}
& d_{i}^{2}=d_{j}^{2}+A B^{2}-2 \cdot d_{j} \cdot A B \cos \alpha \\
& d_{k}^{2}=d_{j}^{2}+A B^{2}-2 \cdot d_{j} \cdot A B \cos \beta
\end{aligned}
$$

where $d_{i}, d_{j}$ and $d_{k}$ values can be calculated from Equation (5). However, $\cos \alpha=-\cos \beta$, hence Equations (15) and (16) can be simplified as

$$
\begin{gathered}
2 A B^{2}=d_{i}^{2}+d_{k}^{2}-2 d_{j}^{2} \\
2(v . \Delta t)^{2}=d_{i}^{2}+d_{k}^{2}-2 d_{j}^{2}
\end{gathered}
$$

Therefore, the value of speed is

$$
v=\frac{\sqrt{\left(d_{i}^{2}+d_{k}^{2}-2 d_{j}^{2}\right)}}{2 \Delta t^{2}}
$$

Now computing the link lifetime $T_{L}$,

$$
T_{L}=T_{d}-T_{E}
$$

where $T_{d}$ represents time taken by node to move from reference point $A$ to point $B$ and $T_{E}$ stands for time elapse by node moving from point $B$ to current position $E$ (Figure 6a). According to the sine rule, it can be obtained as

$$
T_{d}=\frac{R \cdot \sin \theta}{v \cdot \sin \beta}
$$




$$
T_{d}=\frac{2 \Delta t^{2} \cdot R \cdot \sin \theta}{\sin \beta \sqrt{\left(d_{i}^{2}+d_{k}^{2}-2 d_{j}^{2}\right)}}
$$

where $\theta$ represents angle $\angle \mathrm{BSD}$. The value of the node speed and link lifetime are expressed by the above Equations (17) and (18).

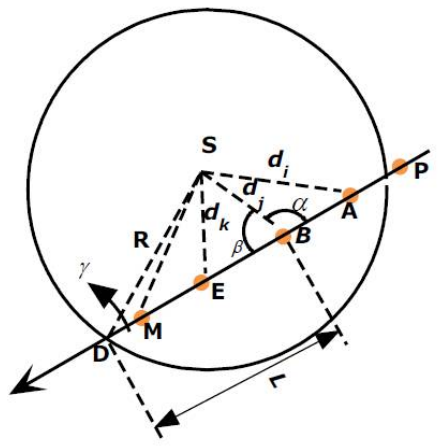

(a)

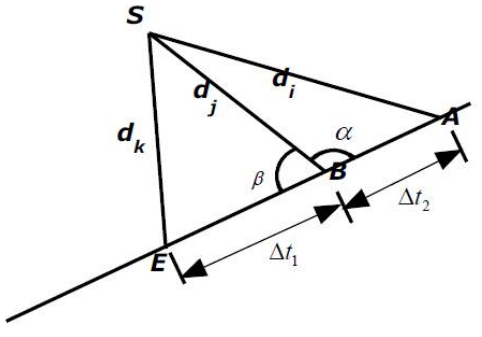

(b)

Figure 6. (a) Node mobility; (b) Mobility estimation.

Unlike AODV, the maintenance operation can be done at local level when link error is encountered which reduces the extra overhead in the network. The proposed approach is based on received signal strength (RSS) to carry out the maintenance at the nearest failure link, which requires keeping two additional entries in the routing table in addition to existing entries (as in AODV protocol, Figure $7 \mathrm{~b}, \mathrm{c}$ ). These entries are the Receive Signal Strength (RSS) of the last node and the RSS difference at the last hop with next node. These values keep on updating at fixed intervals of time as well as compared with the RSS threshold value (THRS). If it is found below the THRS, then a new link is need to be established. Hence, a RMA (Route Maintenance Algorithm) needs to be called in order to remove such an undesirable problem.

In Figure $7 \mathrm{a}$, there is a route $\mathrm{S} \rightarrow \mathrm{C} \rightarrow \mathrm{A} \rightarrow \mathrm{D}$ and link $\mathrm{AD}$ is broken due to erroneous condition. Now, node A (last node) sets the flag = false for route leading from A to D. Furthermore, Node A carries out repair at a local level instead of sending RERR to source. During local repair mechanism, when node $F$ receives RREQ from $A$, send back RREP as it has route to node $D$, now creates a route entry in its routing table with $\mathrm{D}$ as its destination node. However, A does not send RREQ to J and wait till recoil time expires. If node $\mathrm{F}$ is fails to reply within the recoil time then the same steps are repeated with node J till path is repaired. The REPLY message is sent back to the source (S), which contains the number of hop information.

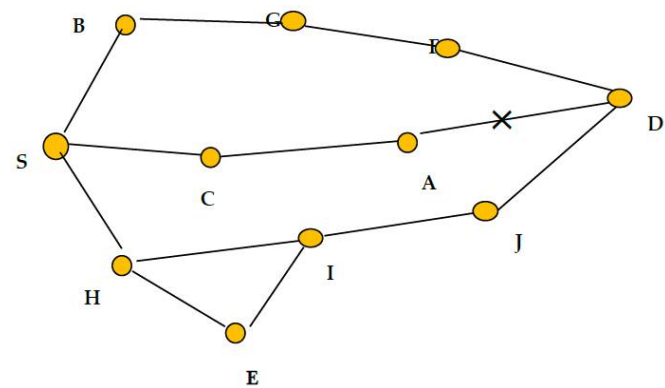

(a)

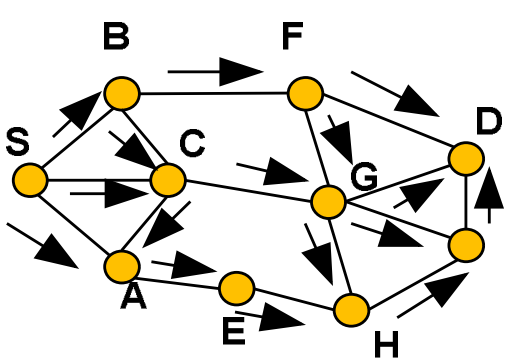

(b)

Figure 7. Cont. 


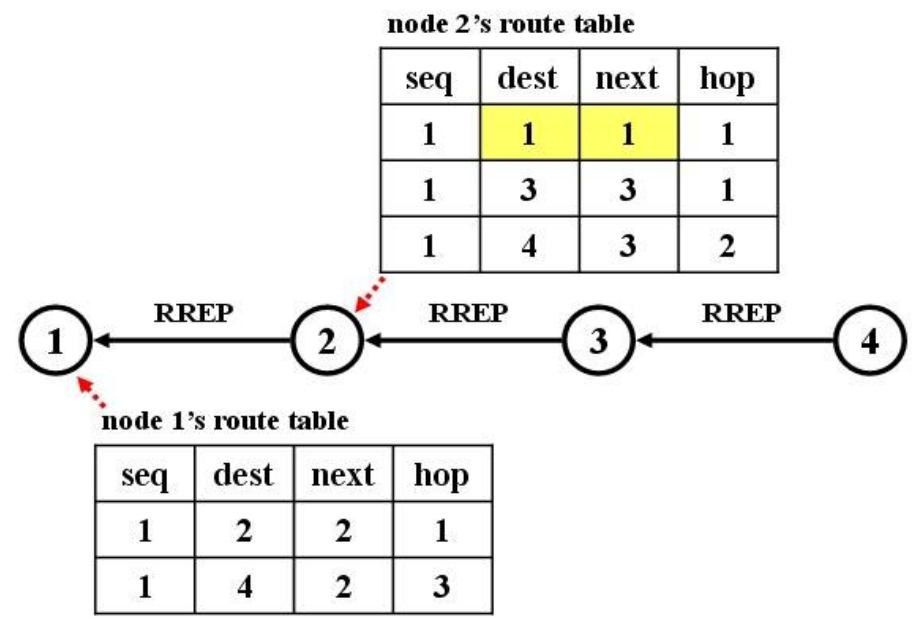

(c)

Figure 7. (a) Illustrates the route maintenance between nodes A and D; (b) Packet transmission from S to $\mathrm{D}$ and table entry with route request in network; (c) Illustrates.

The pseudo code for optimal route discovery is given in Algorithm 3.

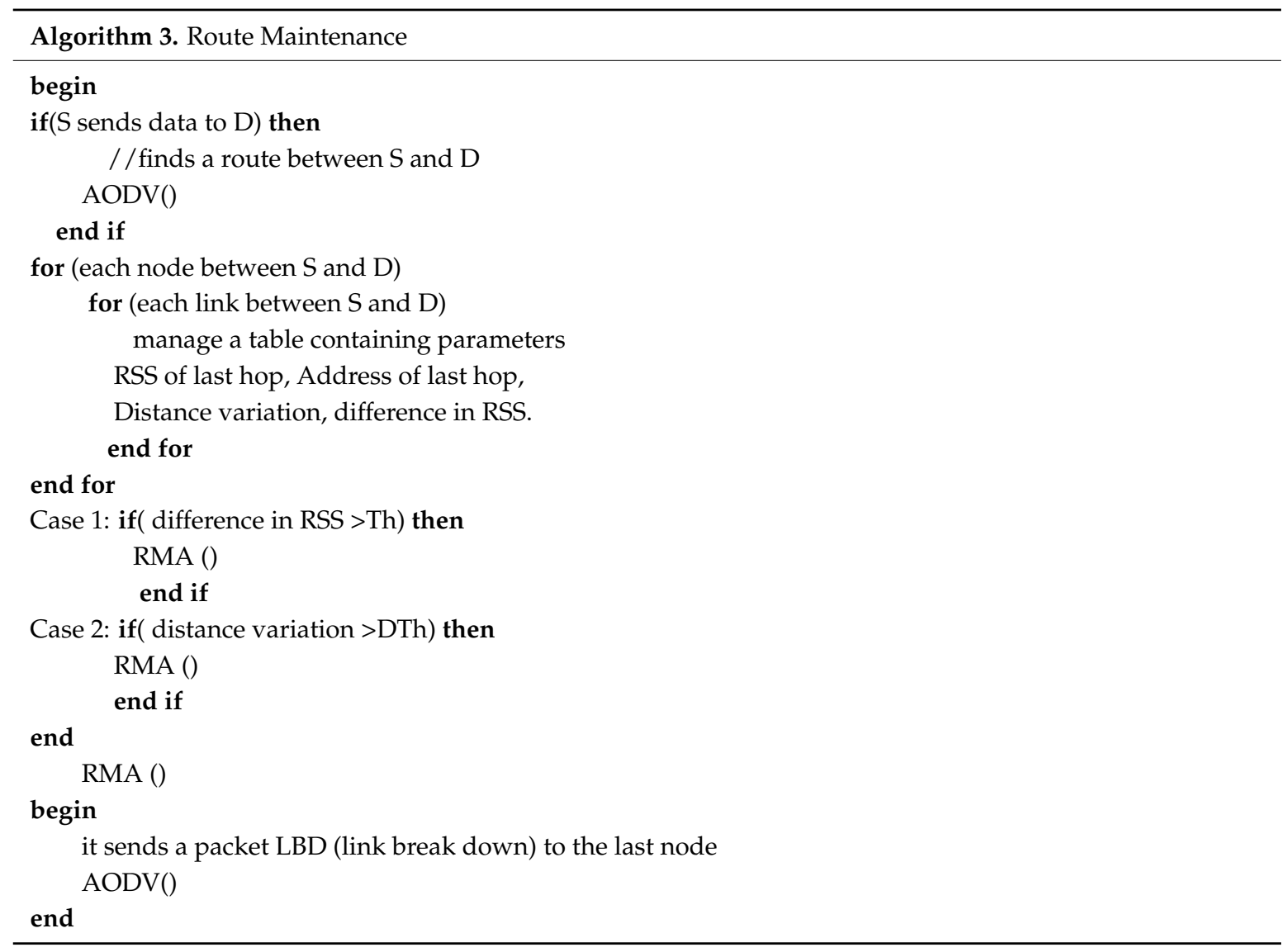

Tables 1-3 show the routing field in routing table for AODV, AOMDV and AOMDV-ER respectively. 
Table 1. Routing Table (AODV).

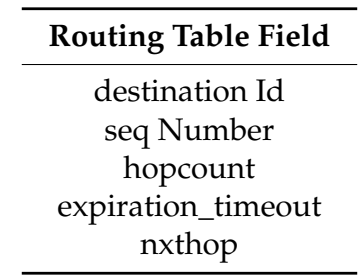

Table 2. Routing Table (AOMDV).

\begin{tabular}{c}
\hline Routing Table Field \\
\hline destination Id \\
Seq_number \\
advertised_hopcount \\
expiration_timeout \\
route_list $\{($ nxthop1, hopcount1), (nxthop2, hopcount2), .. \} \\
\hline
\end{tabular}

Table 3. Routing Table (AOMDV-ER).

\begin{tabular}{c}
\hline Routing Table Field \\
\hline destination Id \\
Seq_number \\
advertised_hopcount \\
route_list\{(nxthop1, hopcount1), (nxthop2, hopcount2),..\} \\
residual energy level \\
threshold energy level \\
receive Signal Strength \\
diff bet RSS values \\
recoiled off time \\
\hline
\end{tabular}

The principal structure of routing table entry in the three mentioned protocols is shown in Table 1. There are few main differences, namely; (i) the hopcount is replaced by advertised hopcount in AOMDV \& AOMDV-ER; (ii) the nxthop is replaced by route list, where AOMDV \& AOMDV-ER keep the nxthop along with the corresponding number of hops in the route and they utilizes multiple path to the destination; (iii) AOMDV-ER has residual energy, threshold energy in order to select the Max-Min path in the network (Figure 2a,b); (iv) furthermore to estimate the link strength receive signal strength and difference between RSS values are kept; and (v) recoiled off time is necessary to limit the number of transmissions.

The function of algorithm has been shown in Figure $6 \mathrm{~b}$ and the corresponding values are depicted in Table 4. The received signal strength (rss_val) is calculated with the help of the signal received by the antenna whereas the recoiled off time (recoil_off_time) is configured just before the execution of the algorithm in the simulator. The threshold energy (thr_eng) of each node (the minimum energy required to transmit a packet to its neighbor) is also set, which helps in the selection of path compared with the residual energy at that instance.

Table 4. Routing table with value (AOMDV-ER).

\begin{tabular}{ccccccccc}
\hline dest_id & seq_no & adv_hpcnt & route_lst & res_eng & thr_eng & rss_val & diff_rss & recoil_off_time \\
\hline D & 11 & 3 & $(\mathrm{~B}, 3)$ & $17 \mathrm{~J}$ & $1 \mathrm{~J}$ & 16 & 1 & $2 \mathrm{~s}$ \\
$\mathrm{D}$ & 11 & 5 & $(\mathrm{~A}, 5)$ & $19 \mathrm{~J}$ & $1 \mathrm{~J}$ & 15 & 2 & $2 \mathrm{~s}$ \\
$\mathrm{D}$ & 11 & 3 & $(\mathrm{C}, 3)$ & $23 \mathrm{~J}$ & $1 \mathrm{~J}$ & 21 & 0 & $2 \mathrm{~s}$ \\
\hline
\end{tabular}




\section{Mathematical Support of Proposed Model for Results}

In order to verify the proposed algorithms for analysis, a set of Lemmas are formulated. These Lemmas are based on system parameters such as protocol overhead, node mobility and packet loss.

\subsection{Lemma-1}

The term mobility refers to the node's average speed during the simulation. The lemma states that the routing overhead increases (in either cases proactive or reactive protocols) with mobility, which can be expressed using Equations (19) and (20).

$$
\begin{aligned}
& \mathrm{M}_{1}>\mathrm{M}_{2}, \varnothing_{\mathrm{P}}\left(\mathrm{M}_{1}\right)>\varnothing_{\mathrm{P}}\left(\mathrm{M}_{2}\right) \\
& M_{1}>M_{2}, \varnothing_{R}\left(M_{1}\right)>\varnothing_{R}\left(M_{2}\right)
\end{aligned}
$$

Proof. Routing overhead is the overhead bear by a protocol to keep updated information about the network. Hence, the number of routing packets is required for the transmission of single data packet. The wired network requires route discovery once and routing overhead incurred only a single time given no mobility in nodes (fixed topology). However, in ad hoc networks, scenario is entirely different as dynamic topology needs frequently route discovery. Additionally, mobility (more frequently changing the topology) of nodes gives rise to multiplier effect to the route discovery and route maintenance means higher mobility, which requires more route discovery, leading to a higher routing overhead.

Suppose each node has probability $p_{r}$ to retransmit the RREQ packet to its destination; let the average destination be $N_{a v g}$ and there are $m$ hops to reach the destination. Hence, the routing overhead for the first hop is $p_{r} \times N_{\text {avg }}$. The overall routing overhead from source to destination is denoted by $\mathrm{R}_{\mathrm{e}}$ and is given by Equation (21).

$$
\begin{gathered}
R_{e}=1+p_{r} \cdot N_{a v g}+p_{r}^{2} \cdot N_{a v g} \cdot N_{f}+p_{r}^{3} \cdot N_{a v g} \cdot N_{f}^{2}+\ldots+p_{r}^{m} \cdot N_{a v g} \cdot N_{f}^{m-1} \\
R_{e}=1+p_{r} \cdot N_{a v g} \sum_{i=0}^{m-1}\left(p_{r} \cdot N_{f}\right)^{i}
\end{gathered}
$$

where $N_{f}$ represent total neighbours number of any node that receive route request packet RREQ, from that node and rebroadcast the same RREQ packet to the next hop with probability $p_{r}$. The Equation (21) is the summation of finite geometric progression, and $R_{e}$ reduces to Equation (22).

$$
R_{e}=\left\{\begin{array}{l}
1+p_{r} \cdot N_{a v g}\left(\frac{1-\left(p_{r} \cdot N_{f}\right)^{m}}{1-p_{r} \cdot N_{f}}\right) \\
1+m \cdot p_{r} . N_{a v g} \text { if } p_{r} \cdot N_{f}=1
\end{array} \text { for any } p_{r} \text { and } N_{f}\right.
$$

Consider the case for reactive routing (e.g., AODV), where intermediate nodes always perform the rebroadcast, hence $\mathrm{p}_{\mathrm{r}}=1$, and we obtain

$$
R_{e}^{A O D V}=\left\{\begin{array}{l}
1+N_{\text {avg }}\left(\frac{1-\left(N_{f}\right)^{m}}{1-N_{f}}\right) \\
1+m \cdot N_{\text {avg }} \text { if } N_{f}=1
\end{array} \text { for any } N_{f}\right.
$$

When mobility of nodes increases, the frequency of route discovery also increases and the value of routing overhead in single route discovery is expressed by Equation (23). Hence it is proved routing overhead increases with mobility of nodes.

In simulation result (Figure 13a), the slope of the tangent line would be the same as the slope of the equation of best fit line on the curve $y=3.72 x+17.2$, whose slope is 3.72 , which is greater than 0 . Hence, the result shows that the routing overhead function is monotonically increasing with the mobility of the node $\square$. 


\subsection{Lemma-2}

The packet loss percentage increases with node mobility in either case, i.e., reactive or proactive protocols, and can be expressed using Equations (24) and (25).

$$
\begin{aligned}
& M_{1}>M_{2}, P L_{P}\left(M_{1}\right)>P L_{P}\left(M_{2}\right) \\
& M_{1}>M_{2}, P L_{R}\left(M_{1}\right)>P L_{R}\left(M_{2}\right)
\end{aligned}
$$

Proof. As per lemma 1, it is stated that the increase in the mobility of that node results in the increase of route discovery process due to the frequent link failure. And link failure improves the chances of packet loss. It is concluded that packet loss percentage increases with mobility.

$M_{1}$ and $M_{2}$ signify two different values of mobility. The derivatives $P L_{P}^{\prime}(M) \geq 0$ and $P L_{R}^{\prime}(M) \geq 0$ show the packet loss function increases with mobility.

In the simulation result (Figure 13b), the slope of the tangent line would be the same as the slope of the equation of best fit line on the curve $y=-1.64 x+98$, which represents the derivative of packet delivery ratio (always inversely proportional to packet loss), whose slope is $-1.64<0$. Hence, the slope of packet loss function would always be greater than 0 . As per the result, it is concluded that the packet loss percentage function is monotonically increasing with the mobility of the node $\square$.

\subsection{Lemma-3}

The energy consumption in routing protocol increases with node mobility in network in both proactive and reactive protocols. It can be represented using Equation (26).

$$
M_{1}>M_{2} \text {, then } E\left(M_{1}\right)>E\left(M_{2}\right)
$$

Proof. The energy consumed by the protocol, the summation of energy consumption in performing route discovery (RD) and energy consumed in routing maintenance (RM), that is, the cost paid in the RD and RM processes, can be illustrated using Equation (18).

$$
E^{p}=E_{R D}+E_{R M}
$$

where $E^{p}$, represents energy consumed in routing protocol, $E_{R D}$ notation used for energy consumed in route discovery process, and $E_{R M}$ stands for route maintenance. The energy in route discovery would be the cost for the RREQ packet and RREP packet during RD process. It can be expressed as below in Equation (28)

$$
\left\{\begin{array}{c}
\sum_{R_{i}=1}^{R_{\max }}\left(E_{R_{i}}\right) R_{i} \quad \text { if no RREP received } \\
E_{\text {rrep }}+\sum_{j=1}^{j=n_{\text {rrep }}}(R R E P)_{j} \quad \text { if } R_{\text {rrep }}=1 \\
\sum_{R_{i}=1}^{R_{\text {rrep }}}\left(E_{R_{i}}\right) R_{i}+\sum_{j=1}^{j=n_{\text {rrep }}}(R R E P)_{j} \quad \text { otherwise }
\end{array}\right.
$$

where, $R_{\text {rrep }}$ and rrep are same and takes the value rrep $=1,23, \ldots$ max. The energy consumption in RM process completion can be expressed as below in Equation (29).

$$
E_{R M}=E_{l-m o n}+E_{L L R}+\sum_{k=0}^{k=j}(R R E P)_{k}
$$

where $E_{l-m o n}$ represents the link monitoring energy consumed in protocol. The total energy consumed can be calculated as the sum of Equations (28) and (29), which is expressed by Equation (27). When the mobility of nodes increases, the routing overhead increases along with corresponding energy consumption in the process. Hence the value of Equation (27) holds the inequality expressed in (26). 
The first order derivative $E^{\prime}(N) \geq 0$, shows that the packet delay function increases with number of nodes. From the result (Figure 13c), the slope of the tangent line would be the same as the slope of the equation of the best fit line curve $y=3.12 x+51.6$, the equation of tangent of the curve. This is 3.12 , a positive value. Here, the energy function is also increasing with mobility of nodes, hence the result is in agreement $\square$.

\section{Results and Discussion}

In this section, we experimentally demonstrate the proposed multipath routing protocol named AOMDV-ER. This protocol is an enhancement of the well-known AOMDV and AOMR-LR routing protocols. The enhancement is observed in terms of network lifetime, routing overheads, PDR and energy consumption over the existing protocols. In this section, we have demonstrated the formally proved Lemmas through experiment. The performance evaluation has been carried out by considering various parameters. The NS2.34 (Snapshot depicted in Figure 8)has been used for the simulation and used random way point model with 150 nodes in an area of $1500 \times 1500 \mathrm{~m}$. Table 5 shows the setting parameters in simulation:

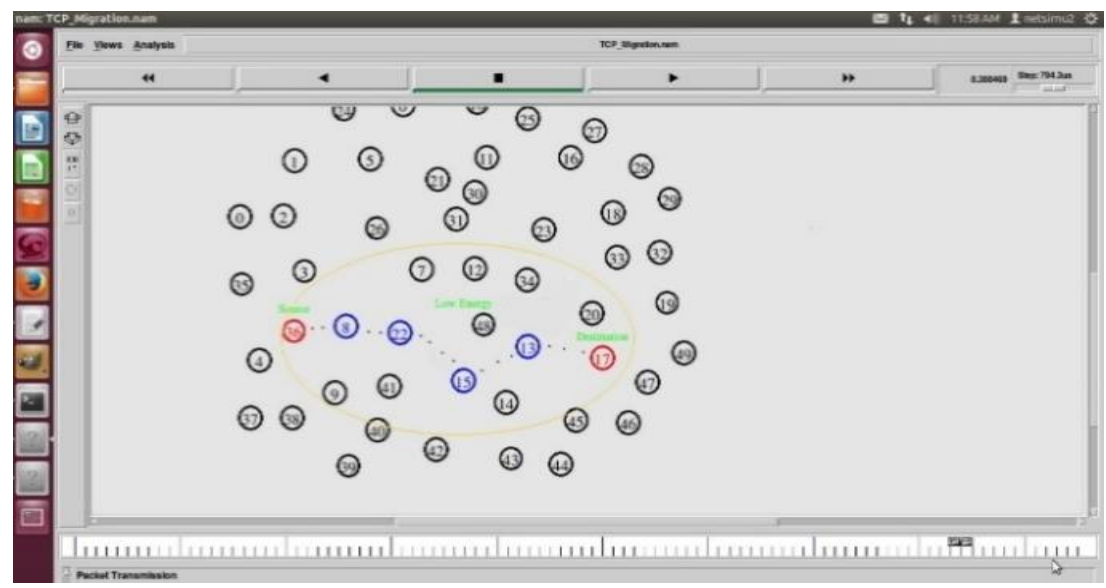

Figure 8. Simulation tool snap shot.

Table 5. Simulation Parameter.

\begin{tabular}{ccc}
\hline Parameter & Value & Unit \\
\hline Number of nodes & 150 & \\
Traffic type & CBR & $\mathrm{m}^{2}$ \\
Area Size & $1500 \times 1500$ & \\
Mobility Model & RWP & $\mathrm{m}$ \\
Transmission range & 500 & Bytes \\
Packet size & 64, 128, 256,512, 1024 & Protocol \\
Routing Protocols & SOMDV-ER, AOMDV, AOMR-LM, & $\mathrm{m} / \mathrm{s}$ \\
Node speed & $0.25,5,7.5,10$ & $\mathrm{~s}$ \\
Simulation Time & $10,20,30,40,50$ & Joules \\
Initial energy & 75 & Joules \\
Transmission energy consumption & 0.02 & Joules \\
Receive energy consumption & 0.01 & Packets \\
Queue size & 50 & \\
Number of runs & 5 &
\end{tabular}




\subsection{Routing Overhead}

Figure 9a represents the deviation in routing overhead's value in AOMR-LM, AOMDV, SRMP and AOMDV-ER. When the node mobility increases like $(0,2.5,5,7.5,10 \mathrm{~m} / \mathrm{s})$, routing overhead of AOMDV increases from $20 \%$ to $70 \%$, AOMR-LM varies from $18 \%$ to $65 \%$, SRMP increases from $25 \%$ to $80 \%$, and our proposed AOMD-ER only increase from $15 \%$ to $54 \%$. The AOMD-ER shows least routing overhead among all because it uses the path which has the strong links where route failure chances is negligible.

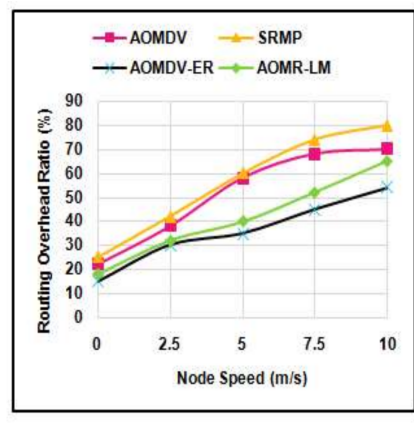

(a)

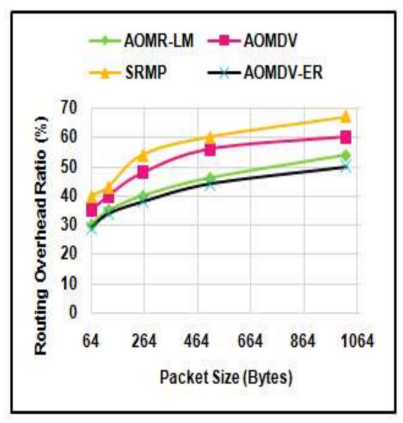

(b)

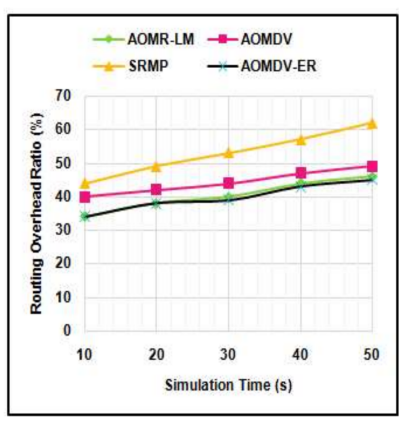

(c)

Figure 9. (a) Overhead vs. Node Speed; (b) Overhead vs. Packet Size; (c) Overhead vs. Sim. time.

Figure $9 \mathrm{~b}$ expresses the variation in routing overhead for AOMR-LM, AOMDV, SRMP and AOMDV-ER. The packet size varies, routing overhead in AOMDV increases from $35 \%$ to $60 \%$, in AOMR-LM varies from $30 \%$ to $54 \%$, in SRMP increases from $40 \%$ to $67 \%$, and our proposed AOMD-ER only increases from $29 \%$ to $50 \%$. The AOMD-ER requires the least routing overhead among all as it required less discovery process along with verification of link stability.

In Figure 9c, the simulation time is varied, the overhead of AOMDV increases from $40 \%$ to $49 \%$, AOMR-LM varies from $34 \%$ to $46 \%$, SRMP increases from $44 \%$ to $62 \%$, and AOMD-ER from $34 \%$ to $45 \%$. The comparison shows that the performance of AOMD-ER is better than other routing protocols.

\subsection{Network Lifetime}

Experimental results of network lifetime have been discussed, for the same protocols. Figure 10a shows the comparison among the network lifetime for the aforementioned protocols. Figure 10b, represents exhausted nodes increases with increasing the packet size. The AOMDV-ER performs better than AOMR-LM because it employs a proficient recoiled node technique.

Figures $10 \mathrm{c}$ and $11 \mathrm{~b}$ show the exhausted node increase with simulation time. The Figure $11 \mathrm{a}, \mathrm{b}$ show the energy consumption with varying simulation time in protocols. The AOMDV-ER performs best because of the recoil node technique, thereby conserving the energy level in nodes while reducing the number of transmission.

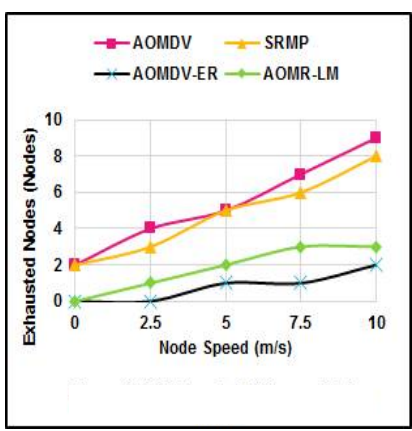

(a)

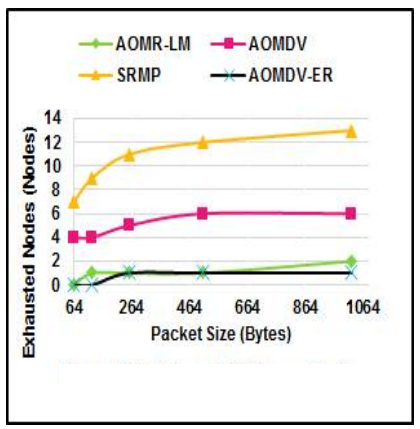

(b)

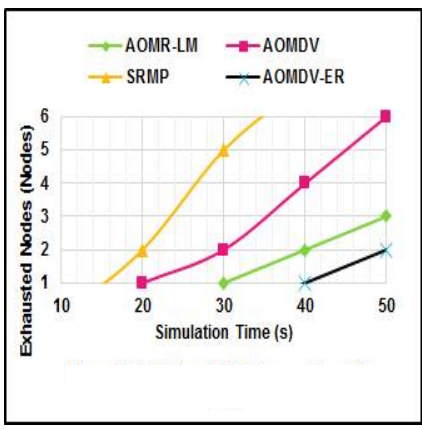

(c)

Figure 10. (a) N Lifetime vs. Node Speed; (b) N. Lifetime vs. Packet Size; (c) N. Lifetime vs. Sim. time. 


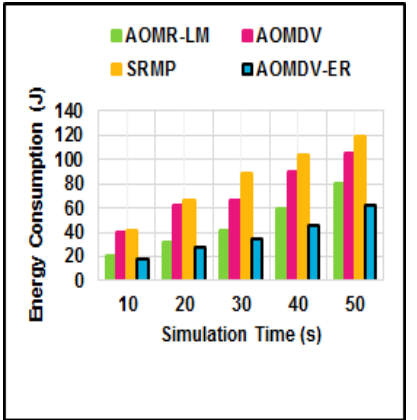

(a)

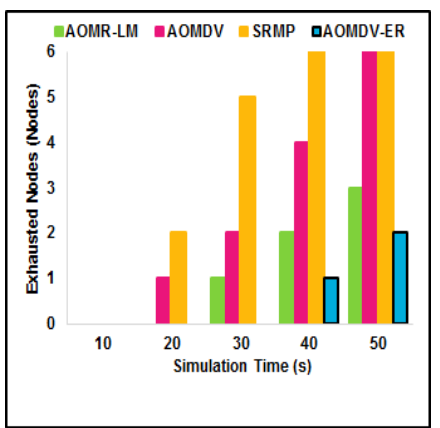

(b)

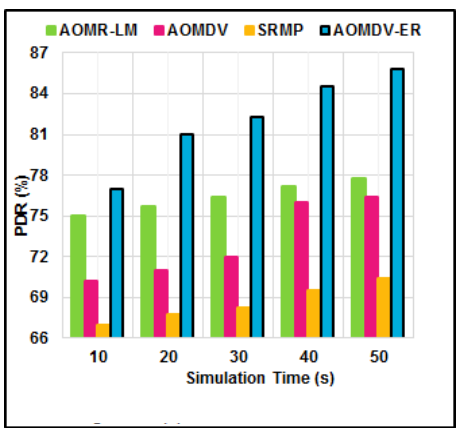

(c)

Figure 11. (a) Energy consumption with time; (b) Exh. nodes with time; (c) PDR with time x.

\subsection{Packet Delivery Ratio}

In Figure 12a, in all protocols the packet delivery ratio (PDR) decreases with mobility; specifically in AOMDV, there is a decrease from 94 to 65\%; in AOMR-LM, a decline from $95 \%$ to $78 \%$; SRMP decreases from $80 \%$ to $47 \%$; and our proposed AOMD-ER decreases from only $97 \%$ to $82 \%$. The AOMD-ER performs better because transmission is on strong and stable link.

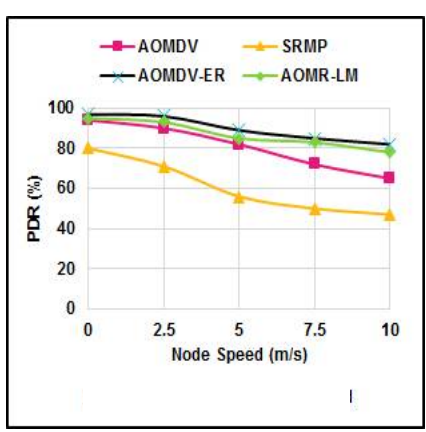

(a)

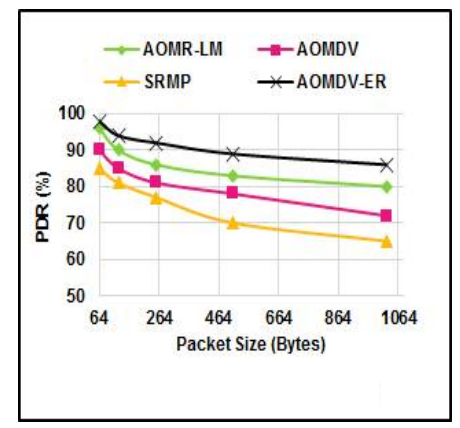

(b)

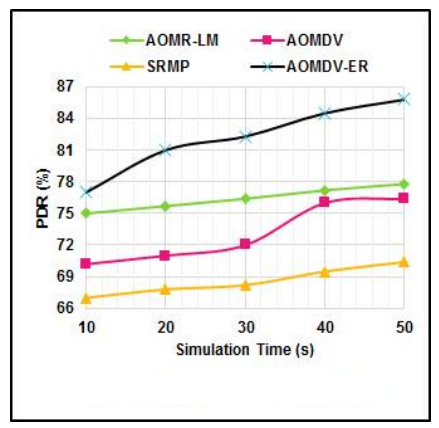

(c)

Figure 12. (a) PDR vs. Node Speed; (b) PDR vs. Packet Size; (c) PDR vs. Sim. time.

Figure $12 \mathrm{~b}$ displays the PDR decreases with packet size. The PDR in AOMDV decreases from $90 \%$ to $72 \%$, AOMR-LM reduces from $96 \%$ to $80 \%$, SRMP decreases from $85 \%$ to $65 \%$ and our proposed AOMD-ER only decreases from $98 \%$ to $86 \%$, due to a smaller drop ratio (using strong link).

Figure 11c, shows PDR increasing with simulation time. The PDR of AOMDV increases from $70 \%$ to $76 \%$, AOMR-LM increases from $75 \%$ to $78 \%$, SRMP increases from $67 \%$ to $70 \%$, and in AOMD-ER from $77 \%$ to $86 \%$. The Figure $11 \mathrm{c}$ shows the PDR\% with varying simulation of time. All these comparisons show that the performance of AOMD-ER is best among all because it uses the stable link nodes and optimal path during transmission.

\subsection{Throughput}

Figure 13a, illustrates the throughput of protocols; it decreases with mobility. In AOMDV, there is a decrease from 1110 to $870 \mathrm{Kbps}$; in AOMR-LM, a reduction from 1120 to $940 \mathrm{Kbps}$; SRMP decreases from 1000 to 665; and the proposed AOMD-ER decreases 1080 to $810 \mathrm{Kbps}$. The AOMR-LM performs best because it requires least time to discover the route even despite the link error encountered.

Figure 13b shows the throughput decreases with packet size. In AOMDV decreases from 1110 to $830 \mathrm{Kbps}$, AOMR-LM reduces from 1117 to $950 \mathrm{Kbps}$, SRMP decreases from 1010 to $720 \mathrm{Kbps}$, and the proposed AOMD-ER only reduces from 1080 to $790 \mathrm{Kbps}$. The AOMR-LM performed better as it requires the least time to discover the route. 
Figure $13 \mathrm{c}$ shows the throughput increasing with the simulation time of the network. The throughput of AOMDV increases from 150 to $980 \mathrm{Kbps}$, in AOMR-LM varies from 180 to $1110 \mathrm{Kbps}$, in SRMP increases from 70 to $651 \mathrm{Kbps}$ and in AOMD-ER from 121 to $801 \mathrm{Kbps}$. The comparison shows that the performance of AOMR-LM is better than other routing protocols because AMOR-LM takes less time in load balancing between high level path and average level during packet transmission.

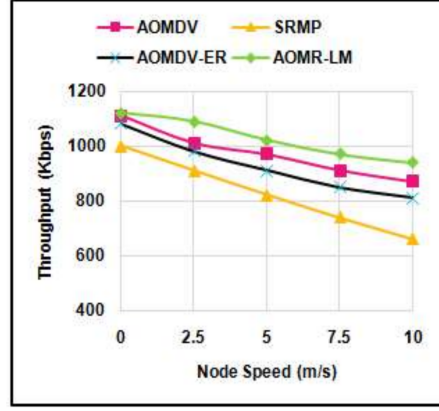

(a)

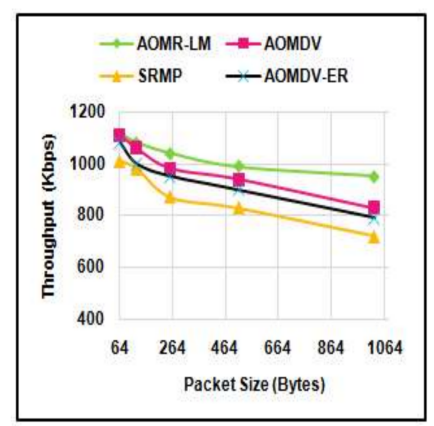

(b)

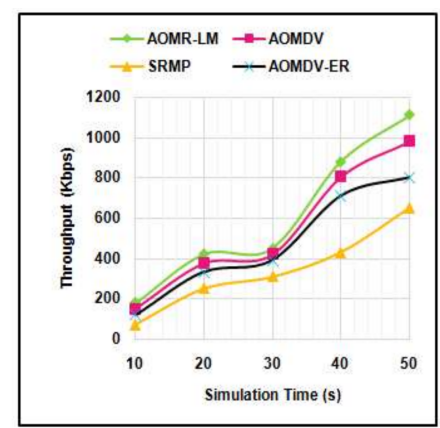

(c)

Figure 13. (a) Throughput vs. Node Speed; (b) Throughput vs. Packet Size; (c) Throughput vs. Sim. time.

\subsection{Energy Consumption}

The experimental results in the figures of energy consumption have been discussed. Figure 14a, expresses the variation of energy consumption in various protocols AOMR-LM, AOMDV, SRMP and AOMDV-ER. In AOMDV, it increases from 70 to 156 Joule; AOMR-LM varies from 60 to $100 \mathrm{~J}$; SRMP increases from 80 to $170 \mathrm{~J}$; and in AOMD-ER, there is an increase from 54 to $86 \mathrm{~J}$. The AOMD-ER outperforms others because in this protocol flooding of packets are limited and only eligible nodes participate in the transmission of packets and remaining nodes wait and sense the network and act only when needed (recoiled technique).

Figure $14 \mathrm{~b}$ illustrates the energy consumption increase with packet size. In AOMR-LM, there is variation from 20 to $80 \mathrm{~J}$; in SRMP, an increase from 42 to $119 \mathrm{~J}$; and in AOMD-ER 18 to $62 \mathrm{~J}$. The AOMD-ER performs best as it conserves energy while restricting flooding and follows the path that has less chance for link error.

Figure $14 \mathrm{c}$ shows the power consumption increases with simulation time. The power consumption in AOMDV increases from 40 to $105 \mathrm{~J}$, AOMR-LM varies from 20 to $80 \mathrm{~J}$, SRMP increases from 42 to $119 \mathrm{~J}$ and AOMD-ER changes from 18 to $62 \mathrm{~J}$. The result shows that performance of AOMD-ER is best among all, as it senses more and transmits less.

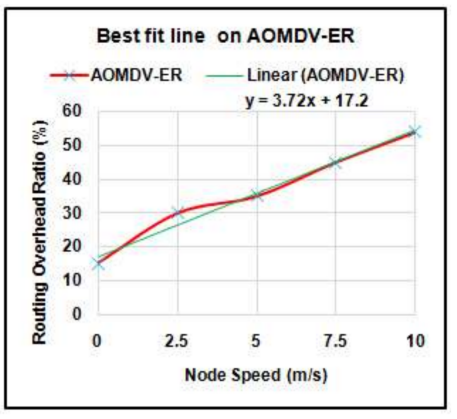

(a)

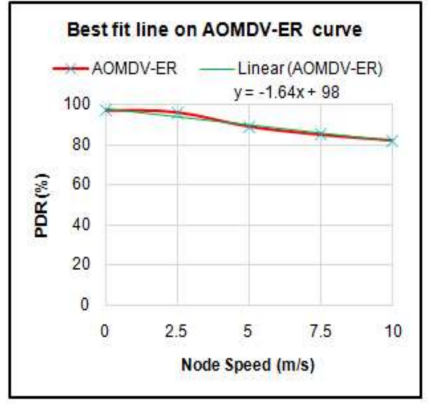

(b)

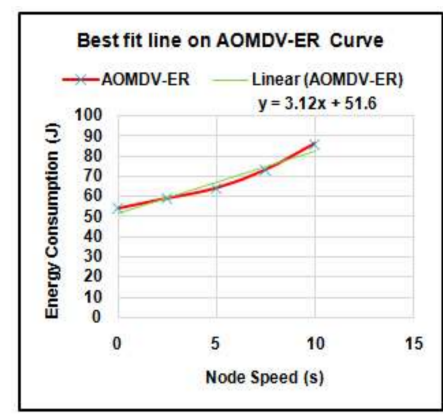

(c)

Figure 14. (a) Best fit line on AODV-ER; (b) Best fit line on AODV-ER; (c) Best fit line on AODV-ER. 
The Experimental results in the figure of energy consumption have been discussed. Figure 14a, expresses the variation of energy consumption in various protocols AOMR-LM, AOMDV, SRMP and AOMDV-ER.

In AOMDV, there is an increase from 70 to 156 Joule; in AOMR-LM, a variation from 60 to $100 \mathrm{~J}$; in SRMP there is an increase from 80 to $170 \mathrm{~J}$; and in AOMD-ER as increase from 54 to $86 \mathrm{~J}$. The AOMD-ER outperforms the others since, in this protocol, flooding of packets is limited and only eligible nodes do transmit the packets and the remaining wait and sense the network and act only when needed (recoiled technique).

Figure 14b illustrates the energy consumption increase with packet size. In AOMR-LM varies from 20 to $80 \mathrm{~J}$, in SRMP increases from 42 to $119 \mathrm{~J}$ and in AOMD-ER 18 to $62 \mathrm{~J}$. The AOMD-ER performs best given it conserves energy while restricting flooding and follows the path that has less chance for link error.

Figure 14c shows the power consumption increase with simulation time. The power consumption in AOMDV increases from 40 to $105 \mathrm{~J}$, in AOMR-LM varies from 20 to $80 \mathrm{~J}$, in SRMP increases from 42 to $119 \mathrm{~J}$ and in AOMD-ER from 18 to $62 \mathrm{~J}$. The result shows that performance of AOMD-ER is best among all, as it senses more and transmits less.

Figure $15 \mathrm{a}-\mathrm{c}$ are also show the energy consumption in the different scenarios, which have already been discussed above.

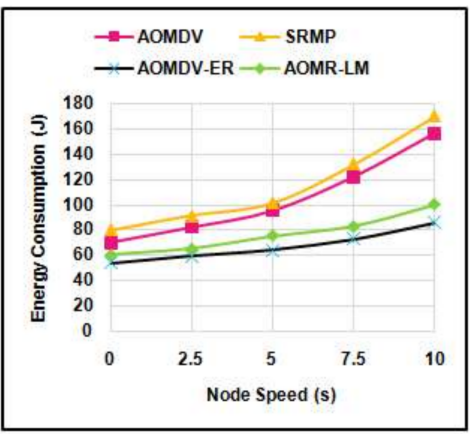

(a)

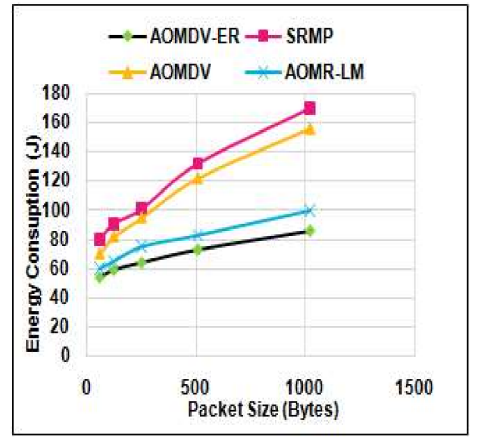

(b)

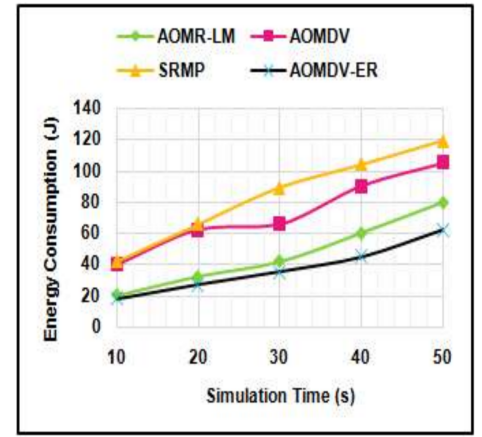

(c)

Figure 15. (a) Energy cons. vs. Node speed; (b) Energy cons. vs. Pack. Size; (c) Energy cons. vs. time.

\section{Conclusions}

The energy reduction multipath routing protocol using recoil technique (AOMDV-ER), ad hoc on demand distance multipath routing with life maximization (AOMR-LM) and ad hoc on demand multipath distance vector routing protocol with fitness function (FF-AOMDV), are all an improvement over the AODV protocol. In this paper, the proposed AOMDV-ER outperforms the existing algorithms, namely AOMR-LM, AOMDV and SRMP, in terms of routing overhead, network lifetime, packet delivery ratio and energy consumption. This is given that in the AOMDV-ER protocol, the nodes perform transmission of packets to destinations smartly using varying recoil off time based on their geographical location. The experimental result shows that AOMDV-ER reduces $22 \%$ and $6 \%$ of the routing overhead, an improvement of $26 \%$ and $33 \%$ of network lifetime, $6 \%$ and $12 \%$ of packet delivery ratio and reduces $14 \%$ and $31 \%$ of power consumption in comparison to AOMDV and AOMR-LM protocols respectively.

Author Contributions: R.K.S. and N.S.C. conceptualized all three algorithms of AOMDV-ER. RKS has carried out the formal analysis of algorithm and software implementation on NS2 simulation tool. R.K.S. created and investigation of all possible scenarios while taking results, whereas the writing review and editing is completed by R.K.S. and N.S.C. together.

Conflicts of Interest: The authors declare no conflict of interest. 


\section{References}

1. Zheng, S.; Weiqiang, W.U.; Zhang, Q. Energy and link-state based routing protocol for MANET. IEICE Trans. Inf. Syst. 2011, 94, 1026-1034. [CrossRef]

2. Rehman, R.A.; Ahmed, S.H.; Kim, B.S. OEFS: On-Demand Energy-Based Forwarding Strategy for Named Data Wireless Ad Hoc Networks. IEEE Access J. 2017, 5, 6075-6086. [CrossRef]

3. Aqeel, T.; Raed, A.; Uddin, M.; Maha, A.; Saba, T. Energy efficient multipath protocol for mobile Ad hoc network using the fitness function. IEEE Access J. 2017, 5, 10369-10382.

4. Aamir, M.; Zaidi, M.A. A buffer management scheme for packet queues in MANET. Tsinghua Sci. Technol. 2013, 18, 543-553. [CrossRef]

5. Sreedevi, M.; Narasimha, C.; Seshadri, R. Efficient Data Delivery over MANET's through Secured EGMP. Adv. Asian Soc. Sci. 2012, 2, 512-516.

6. Lou, W.; Liu, W.; Zhang, Y.; Fang, Y. SPREAD: Improving network security by multipath routing in mobile ad hoc networks. Wirel. Netw. 2009, 15, 279-294. [CrossRef]

7. Yang, S.; Yeo, C.K.; Lee, B.S. Toward reliable data delivery for highly dynamic mobile ad hoc networks. IEEE Trans. Mob. Comput. 2012, 11, 111-124. [CrossRef]

8. Kim, Y.; Ahn, S.; Lee, J. An efficient multicast data forwarding scheme for mobile ad-hoc networks. Information Networking Convergence. In Broadband and Mobile Networking; Springer: Berlin/Heidelberg, Germany, 2005; Volume 1, pp. 510-519.

9. Vaidya, B.; Yeo, S.S.; Choi, D.Y.; Han, S. Robust and secure routing scheme for wireless multihop network. Pers. Ubiquitous Comput. 2004, 13, 457-469. [CrossRef]

10. Biradar, R.; Manvi, S.; Reddy, M. Mesh based multicast routing in MANET: Stable link based approach. Int. J. Comput. Electr. Eng. 2010, 2, 371-380. [CrossRef]

11. Lee, H.O.; Nam, J.S.; Jeon, J.H. Cluster and Location Based Overlay Multicast in Mobile Ad Hoc and Sensor Networks. Int. J. Distrib. Sens. Netw. 2014, 1, 687698. [CrossRef]

12. Kashihara, S.; Hayashi, T.; Taenaka, Y.; Okuda, T.; Yamaguchi, S. Data Delivery Method Based on Neighbor Nodes Information in a Mobile Ad Hoc Network. Sci. World J. 2014, 1, 793171. [CrossRef] [PubMed]

13. Yanbin, Y.A.; Yulin, W.E. A MAC Scheme with QoS Guarantee for MANETs. Int. J. Commun. Netw. Syst. Sci. 2009, 2, 759-763.

14. Moustafa, H.; Labiod, H. A performance comparison of multicast routing protocols in ad hoc networks. In Proceedings of the 14th IEEE Proceedings on Personal, Indoor and Mobile Radio Communications, Beijing, China, 7-10 September 2003; Volume 1, pp. 497-501.

15. Gupta, N.; Gupta, R. LAR-1: Affirmative influences on Energy Conservation and Network Lifetime in MANET. Int. J. Comput. Commun. Control 2014, 9, 284-291. [CrossRef]

16. Kim, J.Y.; Lee, G.M.; Choi, J.K. Efficient multicast schemes using in-network caching for optimal content delivery. IEEE Commun. Lett. 2013, 7, 1048-1051.

17. Sung-Ju, L.; Gerla, M.; Ching-Chuan, C. On Demand Multicast Routing Protocol. IEEE Conf. Wirel. Commun. Netw. 1999, 10, 1298-1302.

18. Jamali, S.; Rezaei, L.; Gudakahriz, S.J. An Energy-efficient Routing Protocol for MANETs a Particle Swarm Optimization Approach. J. Appl. Res. Technol. 2013, 11, 803-812. [CrossRef]

19. Smail, O.; Cousin, B.; Mekki, R.; Mekkakia, Z. A multipath energy conserving routing protocol for wireless ad hoc networks lifetime improvement. EURASIP J. Wirel. Commun. Netw. 2014, 2014, 139-151. [CrossRef]

20. Marina, M.K.; Das, S.R. On-demand multipath distance vector routing in ad hoc networks. In Proceedings of the Ninth International Conference on Network Protocols, Riverside, CA, USA, 11-14 November 2001; pp. 14-23.

(C) 2018 by the authors. Licensee MDPI, Basel, Switzerland. This article is an open access article distributed under the terms and conditions of the Creative Commons Attribution (CC BY) license (http://creativecommons.org/licenses/by/4.0/). 